\title{
On the $L_{\mathrm{x}}-\sigma_{\mathrm{v}}$ relation of groups of galaxies
}

\author{
M. Plionis ${ }^{1,2}$ and H. M. Tovmassian ${ }^{2}$ \\ ${ }^{1}$ Institute of Astronomy \& Astrophysics, National Observatory of Athens, I. Metaxa \& B. Pavlou, P. Penteli 152 36, Athens, \\ Greece \\ e-mail: mplionis@astro.noa.gr \\ 2 Instituto Nacional de Astrofísica Óptica y Electrónica, AP 51 y 216, 72000, Puebla, Pue, México
}

Received 9 April 2003 / Accepted 6 November 2003

\begin{abstract}
We analyse the $L_{\mathrm{x}}-\sigma_{\mathrm{v}}$ relation for the new Mulchaey et al. group Atlas. We find that once we take into account the possible statistical bias introduced by the cutoff in luminosity, we recover a relation that is consistent with that of clusters, i.e., $L_{\mathrm{x}} \propto \sigma^{4.1}$. The larger scatter of this relation for groups of galaxies could be attributed to an orientation effect, due to which the radial velocity dispersion of groups oriented close to orthogonal to the line of sight, would be underestimated. This effect could also contribute to flattening the slope of the group $L_{\mathrm{x}}-\sigma_{\mathrm{v}}$ relation.
\end{abstract}

Key words. galaxies: clusters: general - X-rays: galaxies

\section{Introduction}

Most galaxies in the universe occur in small groups (cf. Geller \& Huchra 1983; Tully 1987; Nolthenius \& White 1987), which in many respects could be considered as poor clusters of galaxies. The detection of X-ray emission from some groups has increased considerably the interest in their study since they have proved to be real entities and not projection effects (for an extensive review see Mulchaey 2000). Solinger \& Tucker (1972) showed that if the source of the X-ray emission is hot gas bound in clusters, then the X-ray luminosity, $L_{\mathrm{X}}$, should be correlated with the optical radial velocity dispersion, $\sigma_{\mathrm{v}}$. Simple theoretical arguments show that in a virialized, isothermal aggregation of gas, which emits thermal bremsstrahlung emission $\left(L_{\mathrm{x}} \propto \int \rho_{\mathrm{gas}}^{2} T(r)^{1 / 2} \mathrm{~d} V\right)$, we can obtain that $L_{\mathrm{x}} \propto M^{4 / 3}$ (where $M$ is the total of the system) and using virial arguments $\left(M \propto \sigma^{3}\right)$ we then have that $L_{\mathrm{x}}$ should be roughly proportional to the fourth power of $\sigma: \log L_{\mathrm{x}} \propto \log \sigma_{\mathrm{v}}^{4}$ (cf. Navarro et al. 1995). Quintana \& Melnick (1982) first showed that the X-ray luminosity of clusters of galaxies obey the expected correlation.

Numerical simulations have shown that the relationship between $L_{\mathrm{x}}$ and $\sigma_{\mathrm{v}}$ for groups should be similar to that of clusters (cf. Navarro et al. 1997), or even steeper if one takes into account radiative cooling which significantly reduces the hot gas fraction at low- $\sigma_{\mathrm{v}}$ (Davé et al. 2002). Such a steep relation for systems with $L_{\mathrm{x}}<10^{43} \mathrm{ergs} \mathrm{s}^{-1}$ has been suggested by Mahdavi \& Geller (2001), although they notice an erratic behavior of the poor groups of galaxies.

Send offprint requests to: $\mathrm{H}$. M. Tovmassian, e-mail: hrant@inaoep.mx
In some observational studies a consistency has been found between the $L_{\mathrm{x}}-\sigma_{\mathrm{v}}$ relation of groups and clusters with a slope 4 (cf. Ponman et al. 1996; Mulchaey \& Zabludoff 1998; Helsdon \& Ponman 2000), while in others a shallower slope has been found, i.e., groups appear to have an enhanced X-ray emission relatively to that predicted from the $L_{\mathrm{x}}-\sigma_{\mathrm{v}}$ relation deduced from clusters of galaxies (cf. Dell 'Antonio et al. 1994; Mahdavi et al. 1997, 2000; Xue \& Wu 2000). Attempts to explain such deviations of the group from the cluster $L_{\mathrm{x}}-\sigma_{\mathrm{v}}$ behaviour have invoked a possible contribution of individual galaxy halos to the group X-ray luminosity (Mahdavi et al. 2000), poorly determined $\sigma_{\mathrm{v}}$ and/or $L_{\mathrm{x}}$ (Zimer et al. 2001) or a large scatter due to a non-equilibrium galaxy velocity distribution (Mahdavi \& Geller 2001).

An alternative explanation, based on a possible orientation effect, was proposed by Tovmassian et al. (2002) (see also Tovmassian et al. 1999; Tovmassian 2002). They considered the relatively small RASSCALS and HCG X-ray group samples (Mahdavi et al. 2000; Ponman et al. 1996, respectively) and showed that the flattening of groups with relatively small velocity dispersions is, on average, larger than those of groups with higher velocity dispersions. They suggested that the shallow shape of $\log L_{\mathrm{x}}-\log \sigma_{\mathrm{v}}$ of groups of galaxies could be partly the result of an underestimation of the velocity dispersion of elongated groups when seen roughly orthogonally to the line of sight, while when seen edge-on, they will have higher $\sigma_{\mathrm{v}}$, probably nearer to their true values. This correlation could be explained if member galaxies in groups move preferentially along the group elongation, since groups have been found to have a prolate-like shape (Hickson et al. 1984; Malykh \& Orlov 1986; Oleak et al. 1998) as in the case of clusters (cf. Plionis et al. 1991). 
Mulchaey et al. (2003) published an X-ray Atlas of groups of galaxies, which is the largest sample of groups studied to date having X-ray ROSAT PSPC pointed observations. In this paper we address two questions: is there sufficient evidence to support claims that groups have enhanced X-ray emission, with respect to clusters (i.e. that the power law index of the $\log L_{\mathrm{x}}-$ $\log \sigma^{\alpha}$ relation has $\alpha \ll 4$ ) and what is the origin of the larger scatter of the $L_{\mathrm{x}}-\sigma$ relation for groups.

\section{Data and results}

In order to address the issue of the possibly enhanced group X-ray emission we have selected from the Atlas of Mulchaey et al. (2003) those objects with detected X-ray emission that contain less than 20 members (since a larger membership should rather define poor clusters). Note that the $L_{\mathrm{x}}$ values were determined after the removal of point sources. We exclude three groups: NGC 2484 and NGC 6251 because they consist of only two members and the NGC 6329 group due to its very large projected length $a(\approx 11 \mathrm{Mpc})^{1}$, which is suspected that it could be a superposition of two or even more groups and hence, its $\sigma_{\mathrm{v}}$ value may be unreliable. We are left with 43 groups in total out of which we have three triplets. Although Focardi \& Kelm (2002) argue that triplets are a distinct class of low- $\sigma_{\mathrm{v}}$ objects, in our case they span the whole $\sigma_{\mathrm{v}}$ range.

In Fig. 1 we plot $\log L_{\mathrm{X}}$ versus $\log \sigma_{\mathrm{v}}$ for the considered groups (open symbols) and for groups with members $N>20$ (squares), which could be considered as poor clusters. The latter are closely located along the line $L_{\mathrm{x}} \propto \sigma_{\mathrm{v}}^{4}$ (solid line).

We perform a direct least-square regression to the $N<20$ groups of the type

$\log L_{\mathrm{x}}=\alpha \log \sigma+C_{1}$

and find a strong correlation with a correlation coefficient $R=0.65$ and a probability of random occurrence $3 \times 10^{-6}$. The fitted parameters are:

$\alpha=1.72 \pm 0.31 \quad C_{1}=37.6 \pm 0.75$.

The determined slope is much shallower than the expected value for clusters $\alpha \simeq 4$, while the value found from the $N>20$ groups is $\alpha \simeq 3.2$. As discussed in the introduction, the shallower slope of the group $L_{\mathrm{x}}-\sigma_{\mathrm{v}}$ relation has already been noticed in other studies as well and has stimulated speculations on the reason for the apparently enhanced X-ray emission of groups. Hence, we could have concluded from our results that this sample supports the claims for a relatively enhanced X-ray emission for groups, but in fact what we are witnessing is the result of a statistical bias.

\subsection{Statistical bias?}

This statistical bias, which resembles the Malmquist bias, appears because of the low- $L_{\mathrm{x}}$ limit (either due to the lower mass limit necessary for the ICM to start emitting in X-rays, or due to the $\mathrm{X}$-ray flux limit in the construction of the sample). This bias is of the same nature as that which enters the scaling relations

\footnotetext{
${ }^{1}$ Lengths are determined assuming $H_{0}=72 \mathrm{~km} \mathrm{~s}^{-1} \mathrm{Mpc}^{-1}$.
}

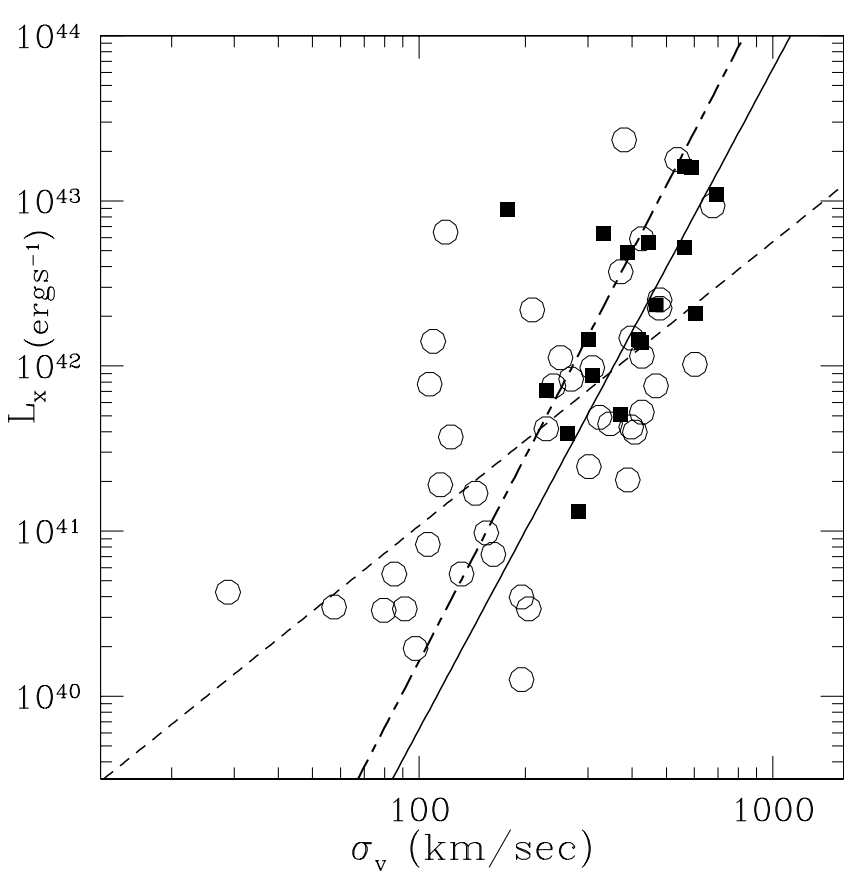

Fig. 1. $L_{\mathrm{x}}-\sigma_{\mathrm{v}}$ relation for groups: those with $N \leq 20$ are represented as circles, while those with $N>20$, supposed to be poor clusters, are marked by filled squares. The solid line is the $\log L_{\mathrm{x}} \propto \log \sigma_{\mathrm{v}}^{4}$ relation of clusters of galaxies. The dashed line is the best direct regression fit. for the groups $\left(\log L_{\mathrm{x}} \propto \log \sigma_{\mathrm{v}}^{1.7}\right)$ and the dot-dashed line is the best inverse regression fit for the groups $\left(\log \sigma \propto(1 / 4.1) \log L_{\mathrm{x}}\right)$.

(e.g. Tully-Fisher, Faber-Jackson, etc.) where the magnitude or flux limit imposes a bias such that the slope of the derived relation is shallower than the nominal one. The larger the scatter of the relation, the larger the bias imposed. This bias may or may not appear in the corresponding cluster relation depending on the amplitude of the scatter around the nominal relation. To understand this bias let us imagine that the cluster relation $L_{\mathrm{x}} \propto \sigma^{4}$ is obeyed by groups as well and let us consider a subset of our sample of groups that have $\sigma_{\mathrm{v}} \simeq 600 \mathrm{~km} \mathrm{~s}^{-1}$ so that they are typically brighter than the lower $L_{\mathrm{x}}$ limit. It is clear that their $\log L_{\mathrm{x}}$ value will be distributed around the mean $\left\langle\log L_{\mathrm{x}}\right\rangle$ value with some dispersion $\sigma$. However, if we take a subsample with $\sigma_{\mathrm{x}} \simeq 100 \mathrm{~km} \mathrm{~s}^{-1}$ so that they are on average quite faint with $\left\langle\log L_{\mathrm{x}}\right\rangle \simeq \log \left(L_{\mathrm{x}}\right)_{\text {limit }}$, then the only groups that will appear in the sample are those with $\left.\log L_{\mathrm{x}}\right\rangle\left\langle\log L_{\mathrm{x}}\right\rangle$ and none with $\log L_{\mathrm{x}}<\left\langle\log L_{\mathrm{x}}\right\rangle$. This will induce the above mentioned bias.

To see this more clearly, we have performed Monte-Carlo simulations in which we assume a relation $L_{\mathrm{x}} \propto \sigma_{\mathrm{v}}^{4}$, and a Gaussian scatter of $\delta \log L_{\mathrm{x}}=0.8$. We then perform a direct (i.e., Eq. (1)) and an inverse least-square regression fit to the resulting data (ie., we fit $\left.\log \sigma_{\mathrm{v}}=(1 / \alpha) \log L_{\mathrm{x}}+C_{2}\right)$. In Fig. 2a we show such a simulation with 5000 "groups", where the solid line is the input $L_{\mathrm{x}} \propto \sigma^{4}$ relation and the dashed line is that recovered from the direct regression. The inverse regression recovers exactly the input slope.

Furthermore, to study more accurately the effect of this statistical bias on our sample we have performed a series of simulations in which we use the observed $L_{\mathrm{x}}$ values of the groups and we derive the values $\sigma_{\mathrm{v}}$, assuming a Gaussian scatter of 


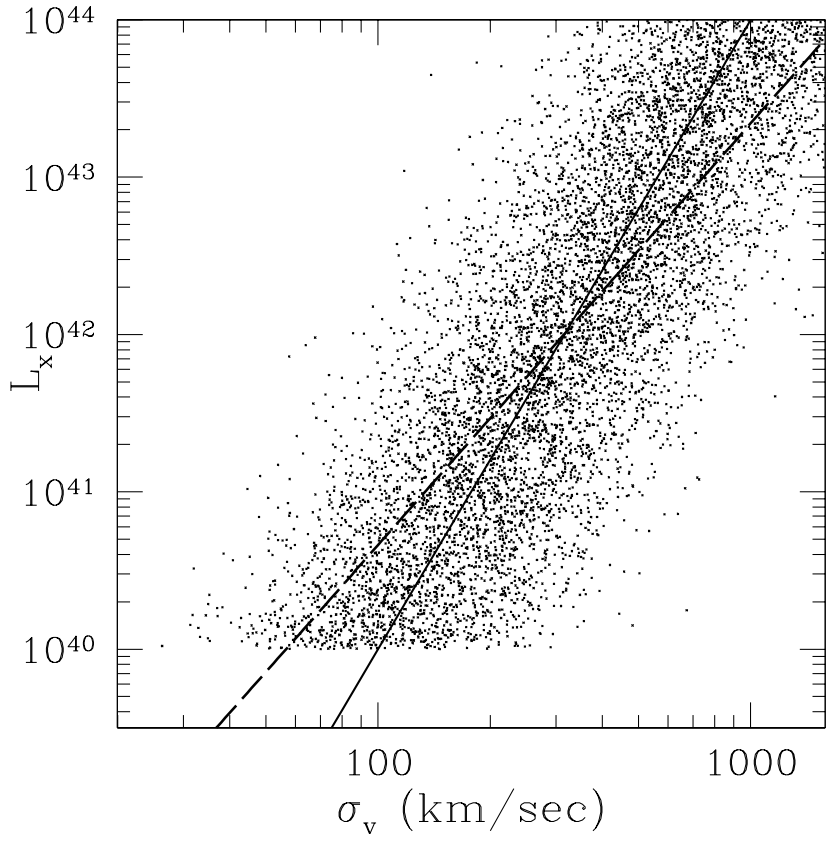

(a)

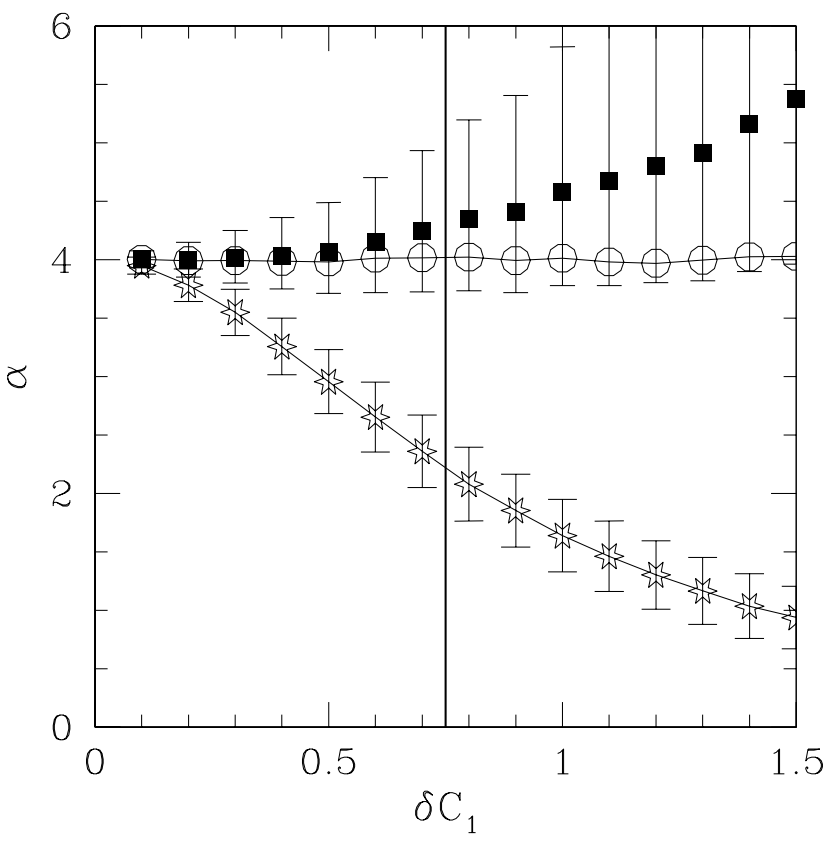

(b)

Fig. 2. a) Manifestation of the statistical bias for 5000 "groups" that have been drawn from a $L_{\mathrm{x}} \propto \sigma_{\mathrm{v}}^{4}$ relation (solid line), which have a scatter of $0.8 \mathrm{in} \log L_{\mathrm{x}}$. The recovered direct regression line is the dashed one. b) Recovered values of the $L_{\mathrm{x}}-\sigma_{\mathrm{v}}$ slope $(\alpha)$ for different amounts of scatter of the relation for the direct regression ( $\log L_{\mathrm{x}} \propto \alpha \log \sigma_{\mathrm{v}}$; star symbols), for the inverse regression $\left(\log \sigma_{\mathrm{v}} \propto(1 / \alpha) \log L_{\mathrm{x}} ;\right.$ open symbols) and for the inverse regression once we have imposed an upper limit of $\sigma_{\mathrm{v}}=1000 \mathrm{~km} \mathrm{~s}^{-1}$.

the $\log L_{\mathrm{x}}-\log \sigma_{\mathrm{v}}$ relation of varying magnitude. We have performed 1000 such simulations for each different value of the Gaussian scatter. In Fig. $2 b$ we plot the derived values of the slope $\alpha$ for both regression methods as a function input scatter. The vertical line indicates the scatter of the relation derived from the $N \leq 20$ sample of groups. The direct regression method (star-like symbols) underestimates severely the input slope, with the underestimation increasing with increasing scatter, while the inverse regression method (open symbols) recovers it accurately. Note also that we plot (squares) the results of the inverse regression in the case of a sharp cutoff at $\sigma_{\mathrm{v}}=1000 \mathrm{~km} \mathrm{~s}^{-1}$, in which case a similar bias, although of smaller magnitude, is introduced in the opposite direction.

It is evident that:

- the inverse regression recovers correctly the input slope of the $\log L_{\mathrm{x}}-\log \sigma_{\mathrm{v}}$ relation for all values of the scatter;

- for the observed amount of scatter $\left(\delta C_{1} \simeq 0.8\right)$ the expected slope of the relation once we use the direct regression is around $\sim 2$, as observed.

As a further test of this bias, we have re-analysed the group data of Xue \& Wu (2000). Their sample consists of 60 groups that have velocity dispersion and X-ray luminosity data. Performing the direct regression fit we recover their results (listed in their Table 3 - OLS method), ie. $\alpha \simeq 1$ and $C_{1} \simeq 40$. However, if we perform an inverse regression fit to their data we recover a very different slope, ie., $\alpha \simeq 6.7$ and $C_{1} \simeq 25.7$. In Fig. 3 we plot the $L_{\mathrm{x}}-\sigma$ correlation for this sample and the fitted lines for both regression methods. Using our Monte-Carlo procedure we have seen that such a dichotomy between the direct and inverse

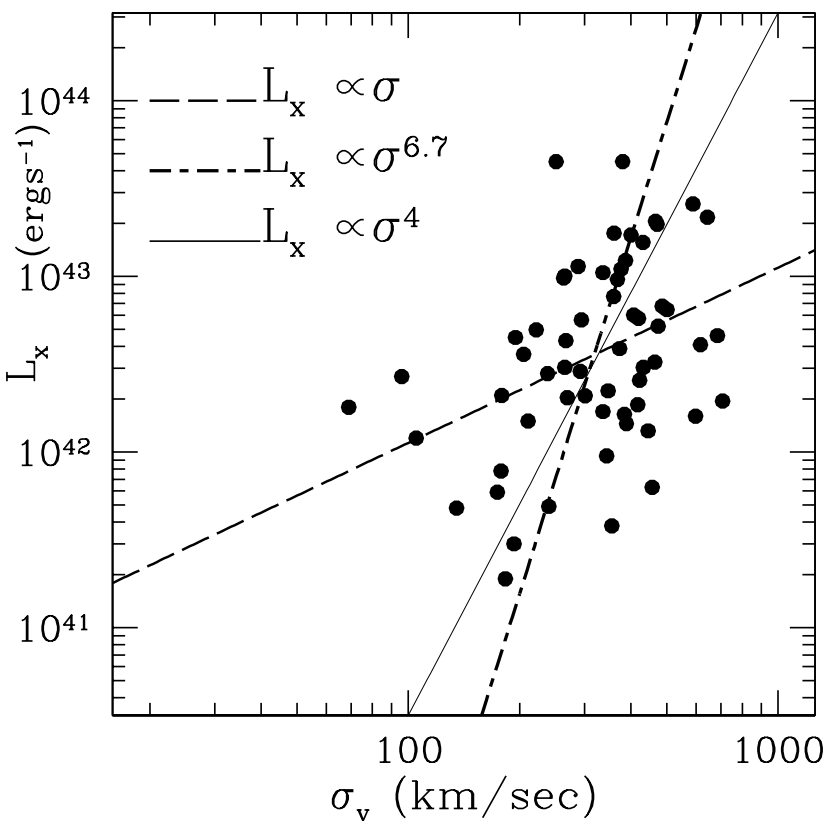

Fig. 3. Results of the Xue \& Wu (2000) sample of groups.

regression methods can be approximately accommodated if there is a scatter of $\sim 1$ in $\log L_{\mathrm{x}}$ and a cutoff at $\sigma \simeq 600 \mathrm{~km} \mathrm{~s}^{-1}$.

Guided by our Monte-Carlo analysis we performed an inverse regression to our sample of $N \leq 20$ groups, and we recovered a slope

$\alpha \simeq 4.1 \pm 0.6$. 
We therefore conclude that this sample of X-ray groups is consistent with the relation found from clusters of galaxies.

\subsection{The scatter in the $L_{x}-\sigma$ relation}

Now we address the issue of the larger scatter of the $L_{\mathrm{x}}-\sigma_{\mathrm{v}}$ relation of groups than of clusters. This is important because, as we have seen previously, the larger scatter will induce an artificial flattening of the $L_{\mathrm{x}}-\sigma_{\mathrm{v}}$ relation.

We show that the larger scatter is not only statistical, due to possible measurement uncertainties especially in the low- $L_{\mathrm{x}}$ and $\sigma_{\mathrm{v}}$ regime, but probably has a large intrinsic component that is due to an orientation effect. We define the axis ratio $b / a$ of the groups studied ${ }^{2}$. The ratio $b / a$ was determined by using the positions of member galaxies mentioned in the corresponding references of Table 1 in Mulchaey et al. (2003). For those groups for which Mulchaey et al. (2003) used the NASA Extragalactic Database (NED), we selected the members of the corresponding groups by their redshifts taking into account the membership number, mentioned in Mulchaey et al. (2003). Note that member galaxies are not drawn from a welldefined and uniform magnitude-limited sample. This can introduce differences in the depth coverage within groups, but such an effect would probably introduce a random error in the determination of the group elongation. In Table 1 we present $b / a$ values for those groups with number of members $N<20$. This is the sample we will investigate in detail.

We noticed that there is a fairly significant correlation ( $R=0.37$ and $\mathcal{P}=0.025$ ) between flattening and velocity dispersion, with flatter systems having lower velocity dispersions (see Fig. 4). Taking the 10 groups with the lowest and the highest values of $\sigma_{\mathrm{v}}\left(\log \sigma_{\mathrm{v}} \leq 2.07\right.$ and $\geq 2.6$, respectively) we find that the median and $68 \%$ quantiles of their $b / a$ ratio are $0.22_{-0.16}^{+0.04}$ and $0.49_{-0.10}^{+0.07}$, respectively.

This difference in the group flattening is also accompanied by a significant difference in the number of group members, with mean values of $\sim 5.2$ and 9.2 respectively. This could be expected either (a) due to the galaxy number - mass correlation (higher number of galaxies implies higher mass which then implies a higher velocity dispersion) or (b) due to the fact that sparser groups are more likely to show, by chance, higher elongations than more dense systems. The latter possibility has been ruled out after performing a large set of MonteCarlo simulations in which we generated the same number of groups and group members as in the two observed subsamples, by randomly placing group members in a sphere and then projecting them onto a plane. The resulting median axial ratio for the low and high- $\sigma$ subsamples was found to be $\sim 0.62 \pm 0.18$ and $\sim 0.7 \pm 0.15$, respectively, significantly larger than the observed values. However, the former possibility cannot explain the significant difference in the flattening of the two extreme

\footnotetext{
${ }^{2} a$ is the angular distance between the most widely separated galaxies in the group, and $b$ is the angular distance of the third galaxy of the group consisting of three galaxies from the line $a$ joining the most separated two galaxies, or is the sum of the angular distances $b_{1}$ and $b_{2}$ of the most distant galaxies on either side of the line $a$ joining the most separated galaxies (Rood 1979).
}

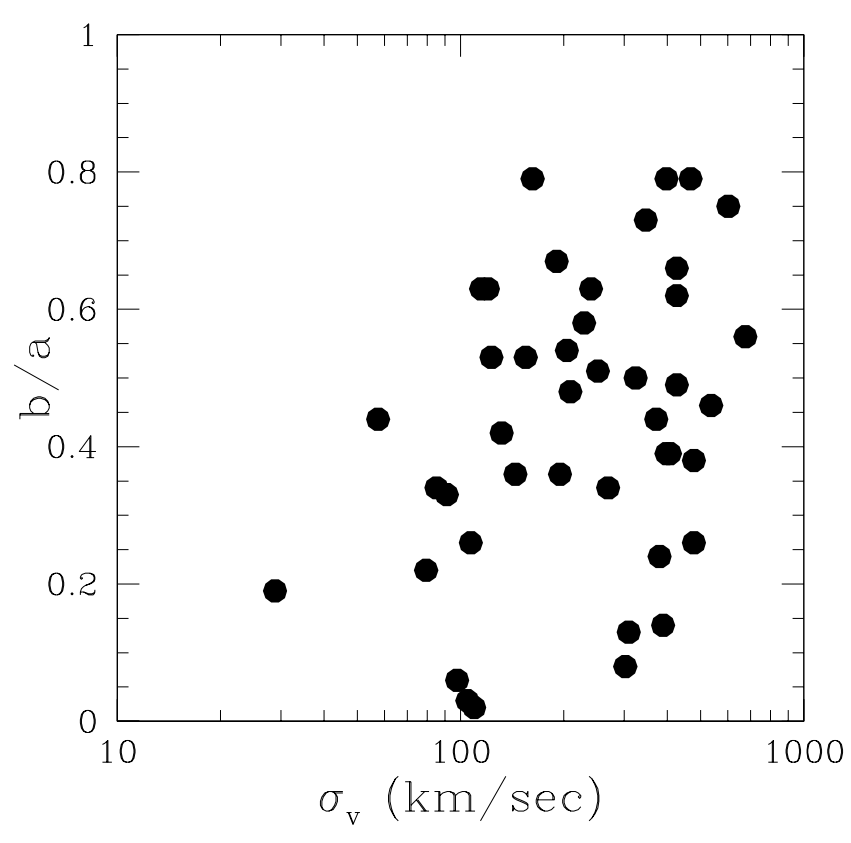

Fig. 4. Group axial ratio versus group $\log \sigma_{\mathrm{v}}$.

subsamples of groups. Why should poorer groups be flatter than richer ones? An explanation could be the different level of group virialization. If groups accrete material anisotropically along one dimensional structures, like filaments, then one may expect that flatter systems are dynamically younger (thus they have lower values of $\sigma_{\mathrm{v}}$ and $L_{\mathrm{x}}$ ) while when virialization takes place, after the groups have accreted enough material, it will drive groups to more spherical configurations, with higher velocity dispersions.

However, this straight-forward explanation cannot account for all the observables. For example, selecting only the groups with $N \leq 5$ members (in total 10 groups), which as expected from the above discussion should be very flat (their median $b / a$ is $0.14_{-0.06}^{+0.05}$ ), we find that although in this subsample there is no correlation of $\sigma_{\mathrm{v}}$ with the number of group members, there is a significant correlation between their flattening and $\sigma_{\mathrm{v}}$ (correlation coefficient $R=0.8$ and $\mathcal{P}_{\text {random }}=0.006$ ), with low- $\sigma_{\mathrm{v}}$ groups being flatter. In the virialization paradigm discussed above such a correlation cannot be explained. To explain such correlations we suggest that an orientation effect is at work, i.e., if galaxy members move along their group major axis (for example, infalling to their common center of mass or rotating in elongated orbits around their gravitational center; cf. Tovmassian 2001, 2002), then flat groups oriented close to orthogonally to the line of sight ( $\operatorname{small} b / a$ ) will have small values of $\sigma_{\mathrm{v}}$, while the opposite is true for flat groups seen edge-on.

According to this view the length of the apparent major axis, $a$, of the flattest groups (small $b / a$ ratio) should also depend on orientation. Groups oriented close to the line of sight will have on average small $a$ and high $\sigma_{\mathrm{v}}$, and groups oriented close to the orthogonal to the line of sight will have large $a$ and small $\sigma_{\mathrm{v}}$. Tovmassian (2002) showed that such anti-correlation between the $a$ and $\sigma_{\mathrm{v}}$ is observed in the case of HCG groups. 
Table 1. The $b / a$ ratios and the number of galaxy members for groups with $2<N<2$.

\begin{tabular}{lrr|lrr|lrl|llr}
\hline \hline Group & $N$ & $b / a$ & Group & $N$ & $b / a$ & Group & $N$ & $b / a$ & Group & $N$ & $b / a$ \\
\hline NGC 315 & 4 & 0.53 & NGC 1407 & 8 & 0.36 & NGC 4065 & 7 & 0.38 & HCG 68 & 5 & 0.53 \\
NGC 326 & 9 & 0.56 & NGC 1587 & 4 & 0.03 & NGC 4104 & 8 & 0.66 & ARP 330 & 8 & 0.44 \\
NGC 524 & 8 & 0.54 & NGC 2300 & 13 & 0.58 & NGC 4125 & 3 & 0.44 & NGC 6338 & 11 & 0.46 \\
HCG 12 & 5 & 0.63 & HCG 37 & 5 & 0.39 & NGC 4261 & 8 & 0.26 & HCG 90 & 16 & 0.67 \\
NGC 720 & 4 & 0.79 & HCG 48 & 3 & 0.08 & SHK 202 & 5 & 0.75 & UGC 12064 & 9 & 0.79 \\
HCG 15 & 6 & 0.49 & CGCG 154-041 & 4 & 0.13 & NGC 4291 & 11 & 0.42 & HCG 92 & 4 & 0.14 \\
HCG 16 & 9 & 0.34 & NGC 3607 & 7 & 0.33 & NGC 4636 & 12 & 0.79 & IC 1459 & 5 & 0.22 \\
UGC 1651 & 3 & 0.02 & NGC 3647 & 6 & 0.24 & NGC 5044 & 9 & 0.63 & NGC 7619 & 7 & 0.51 \\
NGC 1044 & 13 & 0.50 & NGC 3665 & 4 & 0.19 & NGC 5171 & 15 & 0.26 & HCG 97 & 14 & 0.62 \\
IC 1880 & 7 & 0.48 & HCG 57 & 7 & 0.34 & HCG 67 & 14 & 0.73 & NGC 7777 & 4 & 0.63 \\
UGC 2775 & 5 & 0.39 & NGC 3923 & 5 & 0.06 & NGC 5322 & 8 & 0.36 & & \\
\hline
\end{tabular}

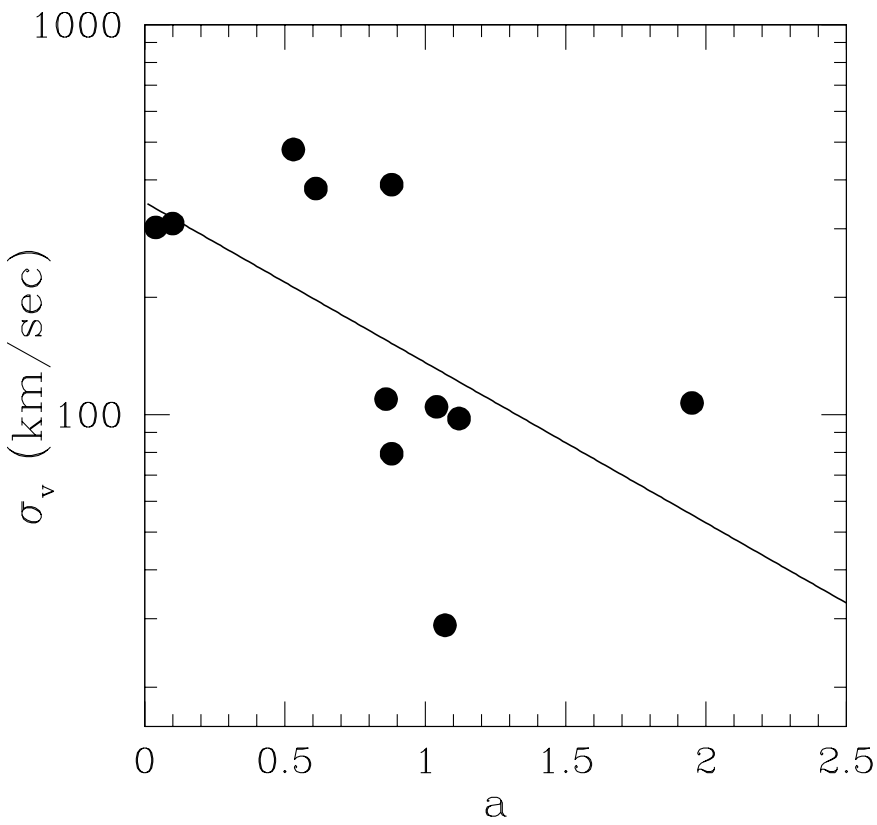

Fig. 5. The flat $(b / a<0.35)$ group $a-\log \sigma_{\mathrm{v}}$ correlation.

To investigate this possibility in our sample, we select the 11 groups with $b / a<0.35$. In Fig. 5 we plot their apparent value of $a$ versus $\sigma_{\mathrm{v}}$. The expected trend is apparent, with $a$ increasing as $\sigma_{\mathrm{v}}$ decreases, and has a probability of occurring randomly of $\mathcal{P}=0.07$. Performing a bootstrap resampling technique to estimate the uncertainty of the above significance level, we find $\delta \mathcal{P}=0.02$.

The existence of the orientation effect does not imply that the virialization arguments, discussed previously, are incorrect. Most probably both are at work since the orientation effect is not apparent in groups with a large number of members, exactly because these groups are richer and most probably in a more advanced dynamical state.

\section{Conclusions}

We have investigated the X-ray luminosity - velocity dispersion relation in the new group Atlas of Mulchaey et al. (2003).
A direct regression shows that there is a strong correlation, with a slope ( $\alpha \simeq 1.7$ ) significantly shallower than that found in clusters of galaxies. However, we attribute this to a statistical bias, resembling the Malmquist bias, that enters in the direct regression approach and which is due to a limit in $L_{\mathrm{x}}$. We have quantified this using Monte-Carlo simulations and once we use the more accurate inverse regression method we obtain a slope of $\sim 4.1$, consistent with the cluster relation. We have also investigated the apparently larger scatter of the $L_{\mathrm{x}}-\sigma$ relation of groups with respect to clusters. We find that at least part of this scatter is intrinsic in nature and due to an orientation effect by which flat groups seen edge-on have their $\sigma_{\mathrm{v}}$ values underestimated. This can be understood by noting that groups of galaxies have a roughly prolate spheroidal shape and thus if member galaxies move along their major axis being accreted for example to their common centre of mass, then the correlation between $\sigma_{\mathrm{v}}$ and their major axis could be due to the different group orientations with respect to the line of sight. Flat groups that are oriented roughly orthogonally to the line of sight will have low values of $\sigma_{\mathrm{v}}$, while when oriented close to the line of sight or at intermediate angles, they will have higher values of $\sigma_{\mathrm{v}}$.

Acknowledgements. We thank the referee for suggestions that helped improve this paper. MP acknowledges funding by the Mexican Government grant No. CONACyT-2002-C01-39679.

\section{References}

Dell'Antonio, I. P., Geller, M. J, \& Fabrikant, D. G. 1994, AJ, 107, 427

Davé, R., Katz, N., \& Weinberg, D. H. 2002, ApJ, 579, 23

Focardi, P., \& Kelm, B. 2002, AA, 391, 35

Geller, M. J., \& Huchra, J. P. 1983, ApJS, 52, 61

Helsdon, S. F., \& Ponman, T. J. 2000, MNRAS, 315, 356

Hickson, P., Ninkov, Z., Huchra, P., \& Mamon, G. A. 1984, in Clusters and Groups of Galaxies, ed. F. Mardirossian, G. Guiricin, \& M. Mezzetti, 367

Mahdavi, A., Böhringer, H., Geller, M. J., \& Ramella M. 1997, ApJ, 483, 68

Mahdavi, A., Böhringer, H., Geller, M. J., \& Ramella, M. 2000, ApJ, 534,114 
Malykh, S. A., \& Orlov, V. V. 1986, Afz 24, 445

Mulchaey, J. S., \& Zabludoff, A. I. 1998, ApJ, 496, 73

Mulchaey, J. S. 2000, ARA\&A, 38, 289

Mulchaey, J. S., Davis, D. S., Mushotzky, R. F., \& Burstain, D. 2003, ApJS, 145, 39

Navarro, J. F., Frenk, C. S., \& White, S. D. M. 1995, MNRAS, 275, 720

Navarro, J. F., Frenk, C. S., \& White, S. D. M. 1997, ApJ, 490, 493

Nolthenius, R., \& White, S. D. M. 1987, MNRAS, 225, 505

Oleak, H., Stoll, D., Tiersch, H., \& MacGillivray, H. T. 1998, Astron. Nachr., 319, 235

Plionis, M., Barrrow, J., \& Frenk, C. 1991, MNRAS, 249, 662

Ponman, T. J., Borner, P. D. J., Ebeling, H., \& Böhringer H. 1996, MNRAS, 283, 690

Ponman, T. J., Cannon, D. B., \& Navarro, J. F. 1999, Nature, 397, 135
Quintana, H., \& Melnick, J. 1982, AJ. 87, 972

Rood, H. J. 1979, ApJ, 233, 21

Solinger, A. B., \& Tucker, W. H. 1972, ApJ, 175, L107

Tovmassian, H. M., Martinez, O., \& Tiersch, H. 1999, A\&A, 348, 693

Tovmassian, H. M., Yam, O., \& Tiersch, H. 2001, Rev. Mex. Astron. Astrofis. 2001, 37, 173

Tovmassian, H. M., Yam, O., \& Tiersch, H. 2002, ApJ, 567, L33

Tovmassian, H. M. 2001, PASP, 113, 543

Tovmassian, H. M. 2002, AN, 323, 488

Tully, R. B. 1987, ApJ, 321, 280

Xue, Y., \& Wu, X. 2000, ApJ, 538, 65

Zimer, M. E., Mulchaey, J. S., \& Zabludoff, A. I. 2002, in Tracing Cosmic Evolution with Galaxy Clusters, ed. S. Borgani, M. Mezzetti, \& R. Valdarnini, ASP Conf. Proc., 268, 459 